\title{
EVALUACIÓN A LOS TRES AÑOS DE EDAD DE UN ENSAYO DE PROGENIES DE Acacia mearnsii DE WILD ESTABLECIDO EN LOS SAUCES, REGIÓN DE LA ARAUCANIA
}

\author{
Pinilla, Juan Carlos $^{8}$; Molina, María Paz ${ }^{9}$; Luengo, Karina ${ }^{10}$ y Navarrete, Mauricio ${ }^{11}$
}

\section{RESUMEN}

Dentro de sus líneas de trabajo el Instituto Forestal (INFOR) está desarrollando diversas investigaciones orientadas a establecer las bases para el uso en plantaciones de algunas especies del género Acacia como alternativas a las especies forestales tradicionalmente empleadas en las plantaciones en el país.

Dentro de este género existen diversas especies forestales de interés comercial, cuya madera presenta usos alternativos y complementarios a las especies tradicionales, agregándose a esto, turnos de cosecha reducidos. Este es el caso de Acacia melanoxylon R.Br., A. mearnsii De Wild. y $A$. dealbata Link.

Las investigaciones realizadas confirman que, desde el punto de vista del crecimiento, de sus aptitudes tecnológicas y sus potencialidades de uso, estas especies se constituyen en una interesante alternativa de producción, lo cual permite orientar a forestadores interesados acerca del cultivo y manejo de este recurso y su potencial productivo. En este contexto y con el fin de mejorar el material de propagación para planes de forestación, se ha establecido en conjunto con la empresa Bosques Cautín SA, un ensayo de progenies (familias) nacionales de Acacia mearnsii en la Región de La Araucanía.

El análisis de la información permitirá establecer el desempeño de árboles seleccionados en el país por sus características de crecimiento y forma, así como proporcionar las primeras orientaciones en relación con las prescripciones de manejo que permitan alcanzar los objetivos de producción.

De los resultados de la investigación realizada previamente se puede concluir que las especies más promisorias en crecimiento y desarrollo son Acacia dealbata y Acacia mearnsii. De igual forma, se han logrado resultados importantes en otra línea, asociada al programa de diversificación forestal, en donde $A$. mearnsii muestra interesantes propiedades para la fabricación de pulpa y para la obtención de taninos desde su corteza.

El presente documento entrega los principales resultados de la evaluación realizada a los 3 años de edad a un ensayo de progenies de Acacia mearnsii, instalado en el año 2011 en el predio San Felipe, de propiedad de Bosques Cautín SA, ubicado en la comuna de Los Sauces, provincia de Malleco, región de la Araucanía.

Palabras clave: Acacia mearnsii, progenies, diversificación de especies.

\footnotetext{
8 Instituto Forestal, Chile, jpinilla@infor.cl

9 Instituto Forestal, Chile, mmolina@infor.cl

${ }^{10}$ Instituto Forestal, Chile, kluengo@infor.cl

${ }^{11}$ Instituto Forestal, Chile, mnavarrete@infor.cl
} 


\section{SUMMARY}

The Chilean forestry Institute (INFOR) is developing several research lines focused on Acacia species in order to establish the necessary information basis to promote the use of some of them in afforestation programs as an alternative to the traditional species used in the country.

Within the Acacia genus there are several species of commercial interest which can produce suitable wood for several uses, complementary to the current used species and under shorter turns. Some of them are Acacia melanoxylon R. Br., A. mearnsii De Wild. and A. dealbata Link.

Different research lines have confirmed that, from the growth, the wood technological properties and the use potentialities points of view, these species represent an interesting productive alternative, so that INFOR, in collaboration with Bosques Cautin SA, has established a Acacia mearnsii national progenies trial at the Araucanía region looking to improve the deployment genetic material for afforestation programs.

The results of the progenies test will allow the behavior evaluation of previously selected trees from different stands in the country according to their growth and shape and will provide valuable information to define some management schemes for different production objectives.

Some previous research results indicate that Acacia dealbata and Acacia mearnsii have a better and promising growth and other research line, related to the national species diversification program, has highlighted interesting properties of $A$. mearnsii to pulp and paper production and to obtain tannins from its bark.

The present a paper shows main results from the Acacia mearnsii progenies test evaluation at 3 years old.

Key words: Acacia mearnsii, progenies, species diversification. 


\section{INTRODUCCIÓN}

La mantención de la variabilidad genética es la clave del mejoramiento genético. El éxito de un programa de mejoramiento depende de la disponibilidad de suficiente variabilidad genética en la población para así poder seleccionar material de propagación en función de caracteres específicos. Esto depende también de la forma en que se ordena y estructura la población para desarrollar el programa a lo largo del tiempo, aspecto fundamental para diseñar la estrategia y llegar a definir los elementos estructurales del ciclo de mejoramiento. En este sentido, el mejorador no solo actuará a través de la selección direccional, sino también estructurando las poblaciones para identificar y manejar la variabilidad.

En el caso de las especies del género Acacia que se cultivan en Chile, los esfuerzos de mejoramiento genético son relativamente recientes, aun cuando el estudio de las mismas cuenta con algunos años de desarrollo. El Instituto forestal consideró a estas especies en su Programa de Introducción de Especies desde los años 60 del siglo pasado, identificando a las especies más promisorias para las condiciones de sitio del país (INFOR, 1998; 1999; Pinilla, 2000).

Diferentes especies de Acacia se están ya usando incipientemente en la forestación en el país, pero para incrementar su uso se requiere conocer los parámetros básicos de crecimiento, productividad y aprovechamiento industrial, lo cual propiciaría la creación de nuevos recursos forestales con ellas, más allá de la áreas habituales de plantaciones en el país, y la consolidación de mercados para sus productos.

Las investigaciones de INFOR en la materia indican que especies del género Acacia que crecen en Chile; como Acacia dealbata Link, Acacia melanoxylon R. Br. y Acacia mearnsii De Wild., en la zona centro sur, y Acacia saligna, en la zona centro norte, han respondido bien a las condiciones edafoclimáticas locales en cada caso, desarrollándose con rapidez y ofreciendo una variedad de usos, lo que las convierte en un recurso económicamente interesante de aprovechar.

Estas características de rápido crecimiento y adaptación a diversos sitios han constituido a estas especies en buenas alternativas también para pequeños y medianos propietarios, quienes podrían obtener ingresos a partir de rodales establecidos en sus predios (Pinilla et al., 2004; Pinilla et al., 2011).

A fines de los años 80, INFOR introduce en la zona central del país Acacia mearnsii, Black Wattle, como parte de las investigaciones realizadas en un proyecto sobre curtientes vegetales, y la especie presenta interesantes resultados iniciales. Debido a estos resultados y la información bibliográfica disponible (Turnbull, 1986; Higa et al., 1998; Kannegiesser, 1990; Searle, 2000; Correa et al., 2011) se ha incorporado la especie al programa de investigación sobre acacias.

Los estudios se amplían, a partir de los 90, con el establecimiento de ensayos de procedencias y, posteriormente, con proyectos de masificación y difusión de las especies, así como con el inicio de estudios de mejoramiento genético y de propiedades de la madera.

Los resultados de la investigación con especies del género Acacia como interesantes alternativas para su uso en forestación, motivó que en 1990 y 1998 se iniciara una serie de ensayos de introducción de procedencias y progenies de acacias australianas con el objetivo de estudiar la adaptación, el crecimiento y el rendimiento de especies y procedencias en distintas situaciones geográficas del país.

Posteriormente y para continuar en esta línea de trabajo, se instalaron nuevos ensayos de Acacia mearnsii, de modo de ampliar el escenario con estas especies, durante los años 2005 y 2006 con progenies (australianas y nacionales) (Cuadro $\mathrm{N}^{\circ} 1$ ). 
Cuadro $\mathrm{N}^{\circ} 1$

ENSAYOS PROCEDENCIAS / PROGENIES Acacia mearnsii ESTABLECIDOS AÑOS 2005 - 2006

\begin{tabular}{|l|c|l|l|l|}
\hline \multicolumn{1}{|c|}{ Ensayo } & $\begin{array}{c}\text { Plantación } \\
\text { (Año) }\end{array}$ & \multicolumn{1}{c|}{$\begin{array}{c}\text { Familias } \\
\left(\mathbf{N}^{\mathbf{0}}\right)\end{array}$} & \multicolumn{1}{|c|}{ Región / Lugar } & \multicolumn{1}{c|}{ Propietario } \\
\hline El Embudo & 2005 & $\begin{array}{l}83 \text { Australianas } \\
21 \text { Nacionales }\end{array}$ & Maule / Retiro & CAF El Álamo \\
\hline Santa Marta & 2005 & 60 Nacionales & Valparaíso / Longotoma & Agrícola Las Loicas \\
\hline Osorno & 2006 & 30 Nacionales & Los Lagos / Osorno & Forestal Anchile \\
\hline
\end{tabular}

Estos ensayos representan la primera base genética de la especie en el país y a esta se suma el ensayo establecido en La Araucanía en 2011. La selección de procedencias y progenies a partir de esta base permitirá producir material de propagación con cierto grado de mejoramiento para incorporar la especie a la forestación en zonas semejantes en el país, para establecer huertos semilleros y también algunas poblaciones de mejoramiento. No obstante estas poblaciones de mejoramiento tendrían que evaluadas genéticamente para dimensionar su variabilidad genética, la cual es posible que deba ser ampliada mediante infusión de nuevos genotipos por medio de la introducción de nuevas procedencias y progenies desde sus áreas de origen en Australia.

El trabajo desarrollado hasta ahora por INFOR con esta especie es un primer esfuerzo por lograr información confiable para iniciar su uso en el país y se ha complementado con estudios de las propiedades de la madera y con los primeros avances hacia un programa de mejoramiento genético.

Estudios realizados por INFOR sobre espaciamiento inicial en Acacia mearnsii abarcando dos áreas geográficas en la región del Bio Bio entregan buenos resultados en cuanto al crecimiento de la especie y dan una primera información sobre rendimientos y crecimientos, antecedentes de importancia para la toma de decisiones por parte de interesados en su cultivo (Pinilla y Navarrete, 2013). Por su adaptabilidad y características, la especie se presenta como una interesante alternativa para la producción de madera, pulpa, biomasa para energía, taninos a partir de su corteza y otros (Pinilla y Navarrete, 2013).

La densidad inicial presentó un efecto significativo sobre el crecimiento en DAP, evidenciándose que a mayor distancia de plantación mayor diámetro medio. Los resultados señalan que las densidades más altas han presentado en general mayores tasas de crecimiento en área basal e igual situación se observó al analizar el estimador de biomasa $D^{2} H$ ), en el cual a una menor distancia de plantación se obtiene una mayor producción. Estos resultados son de interés en función de los productos objetivo que se planteen; para productos menores como pulpa o energía los espaciamientos estrechos aparecen como más convenientes en rotaciones cortas (Pinilla et al., 2013).

La materialización y validación de adecuados modelos predictivos de crecimiento y rendimiento, la ampliación de la base genética representada en el país, la masificación del uso de estas especies junto con el desarrollo de nuevos productos tecnológicos con mayor valor agregado, son actividades que sin duda contribuirán de forma notable a aumentar el interés de empresas y pequeños propietarios por el cultivo de la especie.

\section{OBJETIVO}

Analizar el crecimiento y supervivencia de progenies locales de Acacia mearnsii establecidas en la comuna de Los Sauces, Región de la Araucanía. 


\section{MATERIAL Y MÉTODO}

\section{Área de Ensayo}

El ensayo de progenies de Acacia mearnsii se instaló el año 2011 en el predio San Felipe, de propiedad de Bosques Cautín SA, ubicado en la comuna de Los Sauces, provincia de Malleco, región de la Araucanía, a aproximadamente $6 \mathrm{~km}$ de la ciudad de Los Sauces por el camino a Traiguén.

\section{Instalación del Ensayo}

La preparación previa del terreno consistió en subsolado con bulldozer a una profundidad de $1 \mathrm{~m}$ aproximadamente, quedando camellones cada $3 \mathrm{~m}$. La pendiente del terreno es leve, cercana al $3 \%$ (Figura N 1 ).

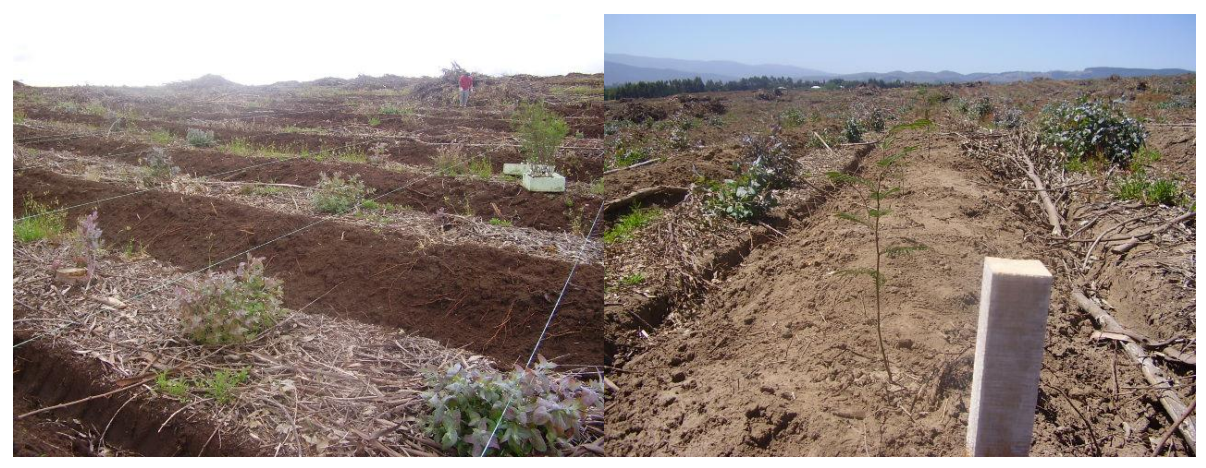

Figura $\mathbf{N}^{\circ} 1$

PREPARACIÓN DE SUELOS Y PLANTACIÓN

\section{Diseño Experimental}

El Ensayo fue establecido en un diseño estadístico de bloques al azar, considerando 25 bloques o repeticiones. En cada bloque se utilizó un diseño experimental de single tree plot (parcela de una planta), con 33 progenies por bloque, utilizando un espaciamiento de $3 \mathrm{~m}$ entre hileras y $2 \mathrm{~m}$ sobre la hilera.

Como elemento de aislación se instaló 1 hilera perimetral de Acacia mearnsii producidas a partir de semilla de procedencia australiana.

Las semillas utilizadas son originarias de árboles plus seleccionados en el país desde diversos ensayos de INFOR, más 2 familias australianas, correspondiendo en total a 33. Las plantas fueron viverizadas en el vivero de INFOR en Concepción.

En el Cuadro $\mathrm{N}^{\circ} 2$ se presenta el listado de progenies utilizadas, en la Figura $\mathrm{N}^{\circ} 2$ se muestra el diseño general del ensayo y en la Figura $N^{\circ} 3$ se muestra una vista general del ensayo en 2014. 
Cuadro $\mathrm{N}^{\circ} 2$

LISTADO DE PROGENIES INCLUIDAS EN EL ENSAYO

\begin{tabular}{|c|l|l|}
\hline Código & Origen (Lugar,Región) & \multicolumn{1}{|c|}{ Tipo de árbol } \\
\hline MR-2 & Antiquina, Cañete, Bío Bío & Árbol BxPxA2 \\
\hline MR-6 & Antiquina, Cañete, Bío Bío & Semillero B1P1A15 \\
\hline MR-7 & Antiquina, Cañete, Bío Bío & Arbol 17 \\
\hline MR-8 & Antiquina, Cañete, Bío Bío & Árbol BxPxA18 \\
\hline MR-10 & Antiquina, Cañete, Bío Bío & Árbol 10 \\
\hline MR-11 & Antiquina, Cañete, Bío Bío & Árbol B1P1A11 \\
\hline MR-14 & Antiquina, Cañete, Bío Bío & Semillero B1P1A14 \\
\hline MR-15 & Antiquina, Cañete, Bío Bío & Semillero B3P14A16 \\
\hline MR-16 & Antiquina, Cañete, Bío Bío & Semillero B3P14A19 \\
\hline MR-18 & Antiquina, Cañete, Bío Bío & Árbol 1 \\
\hline MR-22 & Antiquina, Cañete, Bío Bío & Semillero B1P?A4 \\
\hline MR-26 & Antiquina, Cañete, Bío Bío & Árbol 6 \\
\hline MR-27 & Antiquina, Cañete, Bío Bío & Semillero B4P6A7 \\
\hline MR-28 & Antiquina, Cañete, Bío Bío & Árbol B4P1A8 \\
\hline MR-30 & Mafil, Valdivia, Los Ríos & Plus 2 \\
\hline MR-31 & Mafil, Valdivia, Los Ríos & Plus 3 \\
\hline MR-32 & Mafil, Valdivia, Los Ríos & Plus 4 \\
\hline MR-37 & Antiquina, Cañete, Bío Bío & Árbol 24 \\
\hline MR-40 & San Juan, Nacimiento, Bío Bío & Plus 30 \\
\hline MR-41 & Antiquina, Cañete, Bío Bío & Plus 6 \\
\hline MR-42 & Antiquina, Cañete, Bío Bío & Plus 7 \\
\hline MR-43 & Mafil, Valdivia, Los Ríos & LOTE \\
\hline MR-45 & Australia & 16266 \\
\hline MR-46 & Australia & 14470 \\
\hline MR-49 & Sta. Rosa de Cudá, Florida, Bío Bío & LOTE \\
\hline MR-51 & Rosal, Rere, Bío Bío & Plus 3 \\
\hline MR-52 & Rosal, Rere, Bío Bío & Plus 1 \\
\hline MR-53 & Rosal, Rere, Bío Bío & Plus 5 \\
\hline MR-54 & Mafil, Valdivia, Lo Ríos & Plus 6 \\
\hline MR-56 & Retiro, Maule & Plus 1 \\
\hline MR-59 & Retiro, Maule & Plus 4 \\
\hline MR-60 & Retiro, Maule & LOTE \\
\hline MR-65 & Sta. Rosa de Cudá, Florida, Bío Bío & Plus 3 \\
\hline & & \\
\hline
\end{tabular}




\begin{tabular}{|c|c|c|c|c|}
\hline Bloque 17 & Bloque 18 & Bloque 19 & & \\
\hline Bloque 16 & & Bloque 20 & & \\
\hline \multicolumn{4}{|l|}{ Bloque 15} & \\
\hline \multirow[t]{2}{*}{ Bloque 13} & Bloque 14 & & & \\
\hline & Bloque 10 & Bloque 11 & Bloque 12 & \\
\hline Bloque 23 & Bloque 7 & Bloque 8 & Bloque 9 & \\
\hline Bloque 22 & Bloque 4 & Bloque 5 & Bloque 6 & Bloque 25 \\
\hline Bloque 21 & Bloque 1 & Bloque 2 & Bloque 3 & Bloque 24 \\
\hline
\end{tabular}

Figura $\mathrm{N}^{\circ} 2$

DISEÑO GENERAL DEL ENSAYO DE PROGENIES
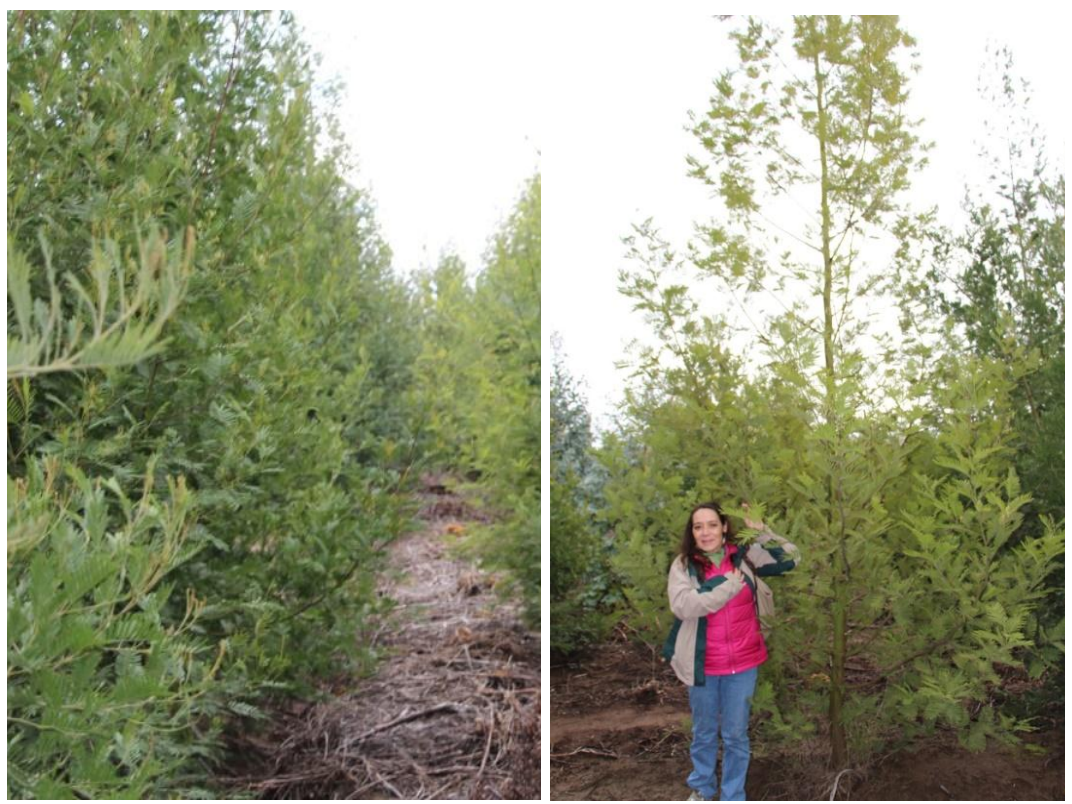

Figura $\mathbf{N}^{\circ} 3$

VISTA GENERAL ENSAYO 3 AÑOS EDAD 


\section{RESULTADOS}

Durante el mes de julio del año 2014 se realizó la segunda evaluación del ensayo de progenies, luego de 33 meses desde su instalación. Esta evaluación se efectuó empleando las variables:
S (\%): Supervivencia
$\operatorname{DAP}(\mathrm{cm})$ :
Diámetro a $1,33 \mathrm{~m}$ de altura
$\mathrm{H}(\mathrm{m})$ : $\quad$ Altura total
$\operatorname{DAP}^{2 *} \mathrm{H}\left(\mathrm{cm}^{3}\right)$ :
Estimador de biomasa

Se efectúa también una comparación con los resultados de la primera evaluación realizada en el año 2013.

\section{Diámetro}

En el Cuadro $\mathrm{N}^{\circ} 3$ se muestra el diámetro alcanzado por las progenies a los 33 meses de edad y en la Figura $\mathrm{N}^{\circ} 4$ se muestran estos resultados gráficamente.

Cuadro $\mathrm{N}^{\circ} 3$

RESULTADOS EN DAP SEGÚN PROGENIES

\begin{tabular}{|c|c|c|c|c|c|}
\hline Progenie & $\begin{array}{c}\text { Media } \\
(\mathrm{cm})\end{array}$ & $\begin{array}{l}\text { Desviación } \\
\text { Estándar }\end{array}$ & $\begin{array}{c}\text { Valor Mínimo } \\
(\mathrm{cm})\end{array}$ & $\begin{array}{l}\text { Valor Máximo } \\
(\mathrm{cm})\end{array}$ & $\begin{array}{c}\text { Mediana } \\
(\mathrm{cm})\end{array}$ \\
\hline 28 & 2,29 & 0,6 & 1,3 & 3,5 & 2,2 \\
\hline 11 & 2,51 & 0,5 & 1,6 & 3,6 & 2,4 \\
\hline 6 & 2,66 & 0,8 & 1,4 & 4,9 & 2,6 \\
\hline 40 & 2,75 & 0,9 & 1,2 & 5,2 & 2,6 \\
\hline 14 & 2,85 & 0,7 & 1,8 & 4,6 & 2,7 \\
\hline 7 & 2,97 & 0,7 & 1,8 & 4,3 & 3,0 \\
\hline 41 & 2,98 & 1,1 & 1,4 & 5,6 & 2,7 \\
\hline 16 & 3,03 & 0,8 & 1,4 & 5,0 & 3,2 \\
\hline 18 & 3,10 & 0,9 & 1,8 & 6,1 & 3,1 \\
\hline 27 & 3,10 & 0,8 & 2,0 & 5,2 & 3,0 \\
\hline 10 & 3,16 & 0,9 & 1,8 & 5,9 & 2,7 \\
\hline 22 & 3,18 & 0,8 & 2,0 & 4,7 & 3,0 \\
\hline 8 & 3,22 & 0,8 & 1,3 & 5,1 & 3,4 \\
\hline 42 & 3,24 & 1,1 & 1,4 & 5,3 & 3,2 \\
\hline 15 & 3,26 & 0,7 & 2,0 & 4,4 & 3,3 \\
\hline 9 & 3,52 & 0,7 & 1,8 & 5,0 & 3,6 \\
\hline 37 & 3,63 & 1,0 & 1,9 & 5,3 & 3,8 \\
\hline 45 & 3,73 & 1,0 & 2,6 & 6,8 & 3,5 \\
\hline 46 & 3,75 & 0,8 & 2,4 & 5,3 & 3,7 \\
\hline 53 & 3,97 & 0,9 & 2,7 & 5,8 & 3,7 \\
\hline 43 & 4,06 & 0,8 & 2,6 & 6,0 & 4,0 \\
\hline 49 & 4,12 & 0,8 & 2,5 & 5,3 & 4,2 \\
\hline 51 & 4,29 & 0,8 & 2,2 & 6,0 & 4,4 \\
\hline 65 & 4,33 & 1,0 & 2,8 & 6,3 & 4,5 \\
\hline 2 & 4,39 & 1,1 & 2,4 & 6,6 & 4,4 \\
\hline 30 & 4,44 & 0,9 & 2,2 & 6,3 & 4,4 \\
\hline 32 & 4,52 & 1,0 & 2,2 & 6,5 & 4,4 \\
\hline 31 & 4,56 & 0,7 & 3,4 & 6,3 & 4,4 \\
\hline 26 & 4,70 & 1,5 & 2,2 & 8,8 & 4,5 \\
\hline 59 & 4,73 & 1,1 & 2,7 & 7,0 & 4,9 \\
\hline 60 & 4,73 & 1,3 & 3,3 & 7,4 & 4,5 \\
\hline 56 & 4,93 & 1,3 & 3,3 & 9,3 & 5,0 \\
\hline 52 & 4,98 & 1,6 & 0,7 & 7,3 & 5,0 \\
\hline 54 & 5,07 & 1,2 & 3,5 & 7,6 & 4,8 \\
\hline
\end{tabular}




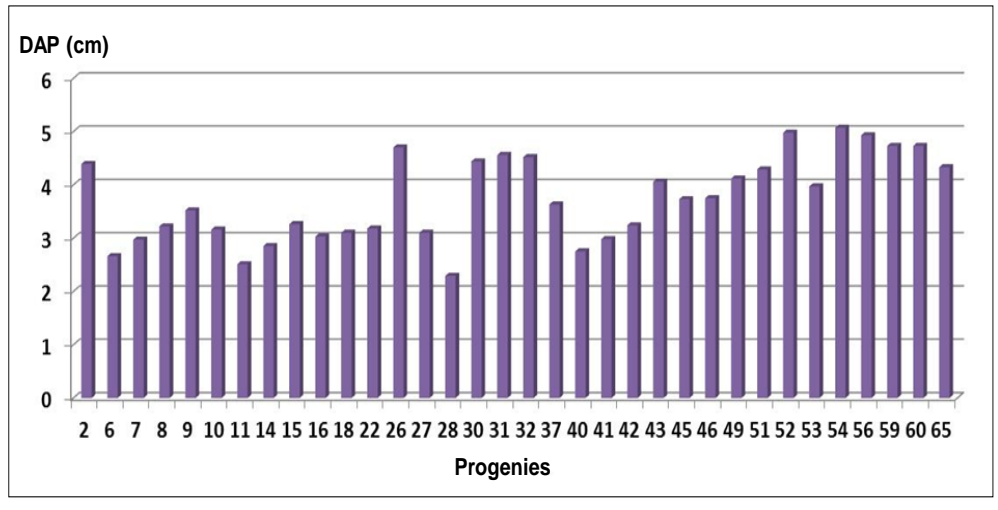

Figura $\mathrm{N}^{\circ} 4$

RESULTADOS EN DAP SEGÚN PROGENIES

En el Cuadro $\mathrm{N}^{\circ} 3$ y en la Figura $\mathrm{N}^{\circ} 4$ es posible apreciar que los mayores valores de DAP medio alcanzan a $5 \mathrm{~cm}$. En la evaluación anterior (2013) a los 21 meses de edad estos eran de $3 \mathrm{~cm}$.

Para analizar estadísticamente los datos se utilizó el software estadístico Infostat ${ }^{\circledR}$. Se realizó un análisis de varianza tradicional (Cuadro $N^{\circ} 4$ ) y, al detectar diferencias significativas, se utilizó el test de comparación múltiple de Tukey (Cuadro $\mathrm{N}^{\circ}$ 5). El modelo utilizado fue:

$$
\begin{aligned}
& \mathbf{Y}=\mathrm{m}+\mathbf{T}+\mathbf{R}+\left(\mathbf{T}^{*} \mathbf{R}\right)+\mathbf{E} \\
& \text { Donde: } \mathrm{m}=\text { Constante } \\
& \mathrm{T}=\text { Tratamiento (Progenies) } \\
& \mathrm{R}=\text { Repetición (Bloques) } \\
& \mathrm{E}=\text { Error }
\end{aligned}
$$

Cuadro $\mathrm{N}^{\circ} 4$

ANÁLISIS DE VARIANZA DAP

\begin{tabular}{|l|r|r|r|r|r|}
\hline \multicolumn{1}{|c|}{ FV } & \multicolumn{1}{c|}{ SC } & gl & CM & F & p-valor \\
\hline Modelo & 598,39 & 783 & 0,76 & 0,73 & 0,7969 \\
\hline Bloque & 65,11 & 24 & 2,71 & 2,58 & 0,0835 \\
\hline Progenie & 226,33 & 33 & 6,86 & 6,52 & 0,0046 \\
\hline Bloque * Progenie & 306,95 & 726 & 0,42 & 0,40 & 0,9885 \\
\hline Error & 8,41 & 8 & 1,05 & & \\
\hline Total & 606,81 & 791 & & & \\
\hline
\end{tabular}

El análisis de varianza señala claramente que existen diferencias significativas entre los valores de DAP obtenido entre las distintas progenies. 
Cuadro $\mathrm{N}^{\circ} 5$

TEST DE COMPARACIÓN DE MEDIAS PARA DAP

\begin{tabular}{|c|c|c|c|c|c|c|c|c|c|c|c|c|c|c|c|}
\hline Progenies & $\begin{array}{c}\text { Medias } \\
\text { (cm) }\end{array}$ & & & & Valc & $\mathrm{rde}$ & Co & npa & aci & & & & & & \\
\hline 28 & 2,29 & A & & & & & & & & & & & & & \\
\hline 11 & 2,51 & A & $B$ & & & & & & & & & & & & \\
\hline 6 & 2,66 & A & B & C & & & & & & & & & & & \\
\hline 40 & 2,75 & A & B & C & $\mathrm{D}$ & & & & & & & & & & \\
\hline 14 & 2,85 & A & $B$ & C & D & & & & & & & & & & \\
\hline 7 & 2,97 & A & B & C & $\mathrm{D}$ & $E$ & & & & & & & & & \\
\hline 41 & 2,98 & A & B & C & $\mathrm{D}$ & $E$ & $\mathrm{~F}$ & & & & & & & & \\
\hline 16 & 3,03 & A & B & C & $\mathrm{D}$ & $E$ & $\mathrm{~F}$ & & & & & & & & \\
\hline 27 & 3,10 & A & B & C & $\mathrm{D}$ & $E$ & $\mathrm{~F}$ & $\mathrm{G}$ & & & & & & & \\
\hline 18 & 3,10 & A & $B$ & C & $D$ & $E$ & $F$ & G & & & & & & & \\
\hline 10 & 3,16 & A & B & C & D & $E$ & $F$ & $\mathrm{G}$ & & & & & & & \\
\hline 22 & 3,18 & $A$ & B & C & $\mathrm{D}$ & $E$ & $\mathrm{~F}$ & $\mathrm{G}$ & & & & & & & \\
\hline 8 & 3,22 & A & $B$ & C & D & $E$ & $\mathrm{~F}$ & $\mathrm{G}$ & $\mathrm{H}$ & & & & & & \\
\hline 42 & 3,24 & A & B & C & $\mathrm{D}$ & $E$ & $F$ & $\mathrm{G}$ & $\mathrm{H}$ & & & & & & \\
\hline 15 & 3,26 & $A$ & $B$ & $\mathrm{C}$ & $\mathrm{D}$ & $\mathrm{E}$ & $\mathrm{F}$ & $\mathrm{G}$ & $\mathrm{H}$ & 1 & & & & & \\
\hline 9 & 3,52 & & B & C & $\mathrm{D}$ & $E$ & $\mathrm{~F}$ & $\mathrm{G}$ & $\mathrm{H}$ & 1 & J & & & & \\
\hline 37 & 3,63 & & & C & $\mathrm{D}$ & $E$ & $\mathrm{~F}$ & G & $\mathrm{H}$ & 1 & J & $\mathrm{K}$ & & & \\
\hline 45 & 3,73 & & & C & D & $E$ & $F$ & $\mathrm{G}$ & $\mathrm{H}$ & I & J & $\mathrm{K}$ & $L$ & & \\
\hline 46 & 3,75 & & & & $\mathrm{D}$ & $E$ & $\mathrm{~F}$ & $\mathrm{G}$ & $\mathrm{H}$ & 1 & $\mathrm{~J}$ & $\mathrm{~K}$ & L & & \\
\hline 53 & 3,97 & & & & & $E$ & $\mathrm{~F}$ & $\mathrm{G}$ & $\mathrm{H}$ & 1 & $\mathrm{~J}$ & $\mathrm{~K}$ & L & & \\
\hline 43 & 4,06 & & & & & & $\mathrm{~F}$ & $\mathrm{G}$ & $\mathrm{H}$ & 1 & $\mathrm{~J}$ & $\mathrm{~K}$ & $\mathrm{~L}$ & & \\
\hline 49 & 4,12 & & & & & & & $\mathrm{G}$ & $\mathrm{H}$ & 1 & $\mathrm{~J}$ & $\mathrm{~K}$ & $\mathrm{~L}$ & & \\
\hline 51 & 4,29 & & & & & & & & $\mathrm{H}$ & 1 & $\mathrm{~J}$ & $\mathrm{~K}$ & $\mathrm{~L}$ & & \\
\hline 65 & 4,33 & & & & & & & & & 1 & $\mathrm{~J}$ & $\mathrm{~K}$ & $\mathrm{~L}$ & & \\
\hline 2 & 4,39 & & & & & & & & & & $\mathrm{~J}$ & $\mathrm{~K}$ & $\mathrm{~L}$ & & \\
\hline 30 & 4,44 & & & & & & & & & & $\mathrm{~J}$ & $\mathrm{~K}$ & $\mathrm{~L}$ & & \\
\hline 32 & 4,52 & & & & & & & & & & $\mathrm{~J}$ & $\mathrm{~K}$ & $\mathrm{~L}$ & & \\
\hline 31 & 4,56 & & & & & & & & & & $\mathrm{~J}$ & $\mathrm{~K}$ & $\mathrm{~L}$ & & \\
\hline 26 & 4,70 & & & & & & & & & & & $\mathrm{~K}$ & $\mathrm{~L}$ & & \\
\hline 59 & 4,73 & & & & & & & & & & & & $\mathrm{~L}$ & & \\
\hline 60 & 4,73 & & & & & & & & & & & & & $M$ & $\mathrm{~N}$ \\
\hline 56 & 4,93 & & & & & & & & & & & & & $M$ & $\mathrm{~N}$ \\
\hline 52 & 4,98 & & & & & & & & & & & & & $M$ & $\mathrm{~N}$ \\
\hline 54 & 5,07 & & & & & & & & & & & & & & $\mathrm{~N}$ \\
\hline
\end{tabular}

\section{Altura}

En el Cuadro $\mathrm{N}^{\circ} 6$ se muestra la altura alcanzada por las progenies a los 33 meses de edad y en la Figura $\mathrm{N}^{\circ} 5$ se muestran estos resultados gráficamente.

Se puede apreciar que los mayores valores en altura a los 33 meses alcanzan a algo más de $6 \mathrm{~m}$. El análisis estadístico se efectuó con el procedimiento ya descrito para el caso del diámetro (Cuadros $\mathrm{N}^{\circ} 7$ y $\mathrm{N}^{\circ} 8$ ). 
Cuadro $\mathrm{N}^{\circ} 6$

RESULTADOS DE ALTURA SEGÚN PROGENIES

\begin{tabular}{|c|c|c|c|c|}
\hline Progenie & $\begin{array}{c}\text { Media } \\
(\mathrm{m})\end{array}$ & $\begin{array}{c}\text { Desviación } \\
\text { Estándar }\end{array}$ & $\begin{array}{c}\text { Valor Mínimo } \\
(\mathrm{m})\end{array}$ & $\begin{array}{c}\text { Valor Máximo } \\
(\mathrm{m})\end{array}$ \\
\hline 28 & 3,8 & 1,08 & 1,4 & 5,7 \\
\hline 40 & 4,2 & 0,84 & 2,5 & 5,4 \\
\hline 11 & 4,2 & 0,71 & 3,3 & 5,7 \\
\hline 14 & 4,3 & 0,56 & 3,4 & 5,3 \\
\hline 6 & 4,4 & 0,81 & 3,0 & 6,2 \\
\hline 41 & 4,4 & 1,00 & 2,8 & 6,7 \\
\hline 7 & 4,6 & 0,57 & 3,2 & 5,7 \\
\hline 16 & 4,6 & 0,80 & 3,3 & 5,9 \\
\hline 8 & 4,7 & 1,07 & 1,6 & 6,3 \\
\hline 18 & 4,7 & 0,87 & 3,1 & 7,2 \\
\hline 15 & 4,7 & 0,82 & 2,8 & 6,0 \\
\hline 27 & 4,8 & 0,84 & 2,9 & 7,4 \\
\hline 22 & 4,8 & 0,78 & 3,2 & 6,4 \\
\hline 42 & 4,9 & 1,12 & 2,8 & 6,7 \\
\hline 45 & 4,9 & 1,01 & 3,5 & 7,1 \\
\hline 9 & 4,9 & 0,89 & 2,6 & 6,9 \\
\hline 10 & 5,0 & 1,01 & 3,7 & 7,9 \\
\hline 37 & 5,0 & 0,95 & 3,4 & 6,6 \\
\hline 46 & 5,1 & 0,87 & 3,1 & 6,9 \\
\hline 43 & 5,3 & 0,81 & 3,2 & 6,5 \\
\hline 53 & 5,3 & 0,81 & 4,1 & 6,8 \\
\hline 2 & 5,5 & 1,10 & 3,4 & 8,3 \\
\hline 49 & 5,5 & 0,89 & 4,0 & 7,2 \\
\hline 65 & 5,7 & 0,89 & 4,4 & 7,0 \\
\hline 51 & 5,8 & 0,77 & 4,1 & 6,9 \\
\hline 60 & 5,9 & 1,07 & 4,8 & 8,7 \\
\hline 30 & 6,0 & 1,10 & 2,6 & 7,8 \\
\hline 31 & 6,0 & 0,72 & 5,0 & 7,3 \\
\hline 32 & 6,0 & 0,76 & 4,5 & 7,5 \\
\hline 26 & 6,0 & 1,09 & 3,9 & 7,6 \\
\hline 59 & 6,1 & 1,02 & 4,1 & 7,8 \\
\hline 56 & 6,1 & 1,07 & 4,4 & 8,0 \\
\hline 52 & 6,2 & 1,35 & 1,5 & 8,0 \\
\hline 54 & 6,3 & 0,94 & 4,7 & 8,0 \\
\hline
\end{tabular}




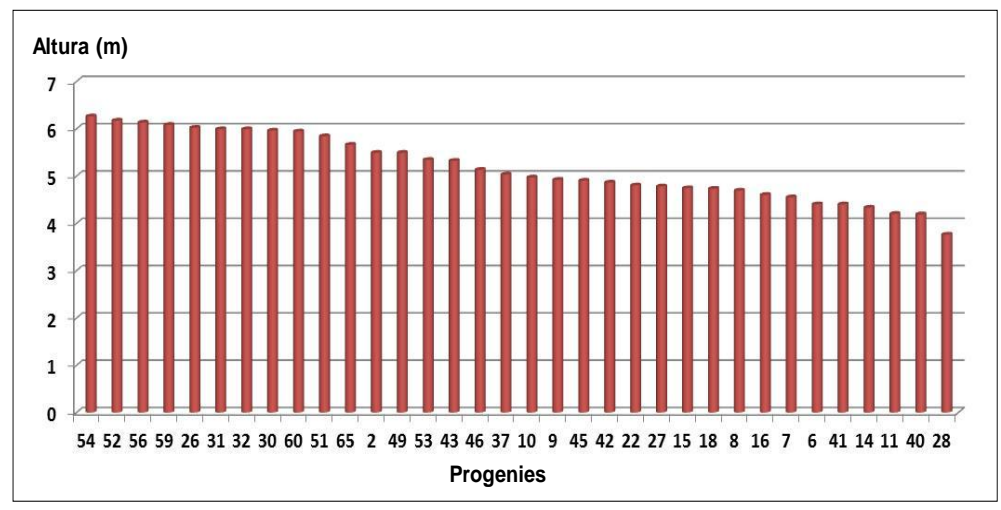

Figura $\mathrm{N}^{\circ} 5$

RESULTADOS EN ALTURA SEGÚN PROGENIES

Cuadro $\mathrm{N}^{\circ} 7$

ANÁLISIS DE VARIANZA ALTURA

\begin{tabular}{|l|r|r|r|r|r|}
\hline \multicolumn{1}{|c|}{ FV } & \multicolumn{1}{c|}{ SC } & \multicolumn{1}{c|}{ gl } & \multicolumn{1}{c|}{ CM } & \multicolumn{1}{c|}{ F } & p-valor \\
\hline Modelo & $1.001,37$ & 789 & 1,27 & 0,49 & 0,9143 \\
\hline Bloque & 363,03 & 33 & 11,00 & 4,23 & 0,0842 \\
\hline Progenie & 135,79 & 24 & 5,66 & 2,18 & 0,2356 \\
\hline Bloque * Progenie & 502,55 & 732 & 0,69 & 0,26 & 0,9953 \\
\hline Error & 10,41 & 4 & 2,60 & & \\
\hline Total & $1.011,77$ & 793 & & & \\
\hline
\end{tabular}

Al igual que en el caso del diámetro, el análisis de varianza señala claramente que existen diferencias significativas entre el valor de las alturas obtenidas por las distintas progenies, por lo que se procedió a hacer el test de comparación de medias.

En la Figura $\mathrm{N}^{\circ} 6$ se muestra la relación DAP $(\mathrm{cm})$ - Altura $(\mathrm{m})$ que se registró para el conjunto de los árboles del ensayo a los 33 meses de edad.

Es posible destacar que hay un crecimiento importante en altura a medida que aumenta el DAP y que existen individuos que a los 33 meses presentan alturas entre los 6 y 9 metros. 
Cuadro $\mathrm{N}^{\circ} 8$

TEST DE COMPARACIÓN DE MEDIAS PARA ALTURA

\begin{tabular}{|c|c|c|c|c|c|c|c|c|c|c|c|c|c|}
\hline Progenies & $\begin{array}{l}\text { Medias } \\
\text { (m) }\end{array}$ & \multicolumn{12}{|c|}{ Valor de Comparación* } \\
\hline 28 & 3,8 & A & & & & & & & & & & & \\
\hline 40 & 4,2 & A & B & & & & & & & & & & \\
\hline 11 & 4,2 & A & B & & & & & & & & & & \\
\hline 14 & 4,3 & A & B & C & & & & & & & & & \\
\hline 6 & 4,4 & A & B & C & & & & & & & & & \\
\hline 41 & 4,4 & A & B & C & & & & & & & & & \\
\hline 7 & 4,6 & A & B & C & D & & & & & & & & \\
\hline 16 & 4,6 & A & B & C & D & & & & & & & & \\
\hline 8 & 4,7 & A & B & C & D & $E$ & & & & & & & \\
\hline 18 & 4,7 & A & B & C & D & $E$ & & & & & & & \\
\hline 15 & 4,7 & A & B & C & D & $E$ & & & & & & & \\
\hline 27 & 4,8 & A & B & C & D & $E$ & & & & & & & \\
\hline 22 & 4,8 & A & B & C & D & $E$ & & & & & & & \\
\hline 42 & 4,9 & & B & C & D & $\mathrm{E}$ & $\mathrm{F}$ & & & & & & \\
\hline 45 & 4,9 & & B & C & D & $E$ & $\mathrm{~F}$ & $\mathrm{G}$ & & & & & \\
\hline 9 & 4,9 & & B & C & D & $E$ & $F$ & $\mathrm{G}$ & $\mathrm{H}$ & & & & \\
\hline 10 & 5,0 & & $\mathrm{~B}$ & C & D & $E$ & $\mathrm{~F}$ & $\mathrm{G}$ & $\mathrm{H}$ & I & & & \\
\hline 37 & 5,0 & & $\mathrm{~B}$ & C & D & $E$ & $\mathrm{~F}$ & $G$ & $\mathrm{H}$ & I & J & & \\
\hline 46 & 5,1 & & $\mathrm{~B}$ & C & D & $\mathrm{E}$ & $\mathrm{F}$ & $\mathrm{G}$ & $\mathrm{H}$ & I & J & K & \\
\hline 43 & 5,3 & & & C & D & $E$ & $\mathrm{~F}$ & $\mathrm{G}$ & $\mathrm{H}$ & I & $\mathrm{J}$ & $\mathrm{K}$ & L \\
\hline 53 & 5,3 & & & C & D & $\mathrm{E}$ & $\mathrm{F}$ & $\mathrm{G}$ & $\mathrm{H}$ & I & $\mathrm{J}$ & $\mathrm{K}$ & L \\
\hline 49 & 5,5 & & & & D & $E$ & $\mathrm{~F}$ & $\mathrm{G}$ & $\mathrm{H}$ & I & $\mathrm{J}$ & $\mathrm{K}$ & L \\
\hline 2 & 5,5 & & & & D & $E$ & $\mathrm{~F}$ & $\mathrm{G}$ & $\mathrm{H}$ & I & $\mathrm{J}$ & K & L \\
\hline 65 & 5,7 & & & & & $E$ & $\mathrm{~F}$ & $G$ & $\mathrm{H}$ & I & $\mathrm{J}$ & $\mathrm{K}$ & L \\
\hline 51 & 5,8 & & & & & & $\mathrm{~F}$ & $\mathrm{G}$ & $\mathrm{H}$ & I & $\mathrm{J}$ & $\mathrm{K}$ & L \\
\hline 60 & 5,9 & & & & & & & $\mathrm{G}$ & $\mathrm{H}$ & 1 & $\mathrm{~J}$ & $\mathrm{~K}$ & L \\
\hline 30 & 6,0 & & & & & & & & $\mathrm{H}$ & I & $\mathrm{J}$ & $\mathrm{K}$ & L \\
\hline 31 & 6,0 & & & & & & & & & 1 & $\mathrm{~J}$ & $\mathrm{~K}$ & L \\
\hline 32 & 6,0 & & & & & & & & & & $\mathrm{~J}$ & $\mathrm{~K}$ & L \\
\hline 26 & 6,0 & & & & & & & & & & $\mathrm{~J}$ & $\mathrm{~K}$ & L \\
\hline 59 & 6,1 & & & & & & & & & & & $\mathrm{~K}$ & L \\
\hline 56 & 6,1 & & & & & & & & & & & $\mathrm{~K}$ & L \\
\hline 52 & 6,2 & & & & & & & & & & & & L \\
\hline 54 & 6,3 & & & & & & & & & & & & L \\
\hline
\end{tabular}

${ }^{*}$ Letras distintas indican diferencias significativas $(p<=0,05)$ 


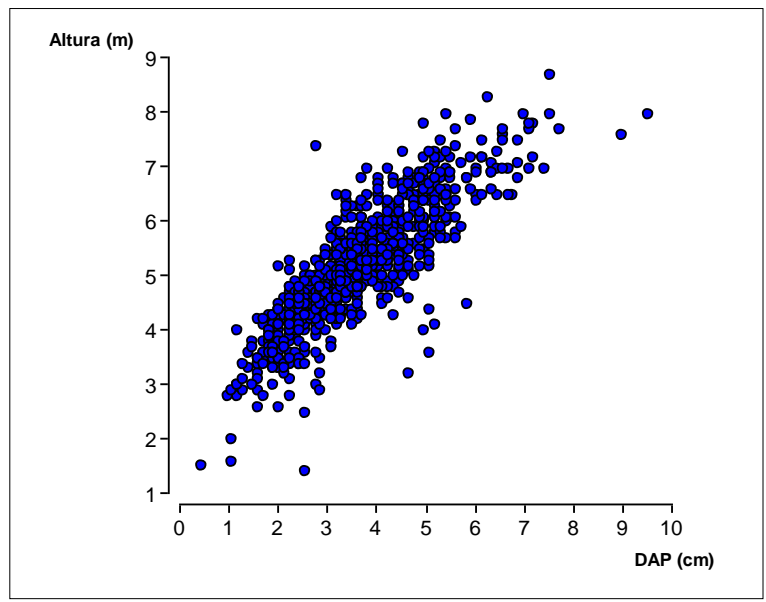

Figura $\mathrm{N}^{\circ} 6$

Relación DAP-Altura

\section{Estimación de Biomasa}

Como una forma de estimar la biomasa resultante por progenie, se utilizó el estimador de biomasa habitualmente empleado en estas comparaciones dado por el cuadrado del DAP multiplicado por la altura $\left(D^{2} \mathrm{H}\right)$ expresado en $\mathrm{cm}^{3}$.

Este resultado se indica en el Cuadro $N^{\circ} 9$ y en forma gráfica en la Figura $N^{\circ} 7$.

Para el análisis estadístico se usó el procedimiento ya indicado para las variables respuesta anteriores (Cuadros $\mathrm{N}^{\circ} 10$ y $\mathrm{N}^{\circ} 11$ ).

Al igual que en las variables anteriores, el análisis de varianza señala claramente que existen diferencias significativas entre el valor de la estimación de biomasa obtenido entre las distintas progenies, por lo que se efectúa la correspondiente comparación de medias. 


\section{Cuadro $\mathrm{N}^{\circ} 9$}

RESULTADOS DE BIOMASA SEGÚN PROGENIES

\begin{tabular}{|c|c|c|c|c|}
\hline Progenie & $\begin{array}{c}\text { Media } \\
\left(\mathrm{cm}^{3}\right)\end{array}$ & $\begin{array}{l}\text { Desviación } \\
\text { Estándar }\end{array}$ & $\begin{array}{c}\text { Valor } \\
\text { Mínimo } \\
\left(\mathrm{cm}^{3}\right)\end{array}$ & $\begin{array}{c}\text { Valor } \\
\text { Máximo } \\
\left(\mathrm{cm}^{3}\right)\end{array}$ \\
\hline 28 & 2.349 & $1.837,59$ & 338 & 6.589 \\
\hline 11 & 2.866 & $1.527,18$ & 922 & 6.221 \\
\hline 6 & 3.665 & $3.231,76$ & 588 & 14.886 \\
\hline 40 & 3.695 & $2.738,05$ & 403 & 11.086 \\
\hline 14 & 3.936 & $2.501,14$ & 1.102 & 10.580 \\
\hline 7 & 4.429 & $2.490,3$ & 1.037 & 10.055 \\
\hline 16 & 4.835 & $3.154,96$ & 784 & 14.750 \\
\hline 41 & 4.972 & $4.669,47$ & 549 & 17.875 \\
\hline 27 & 5.208 & $3.613,19$ & 1.520 & 15.954 \\
\hline 18 & 5.396 & $5.165,09$ & 1.004 & 26.791 \\
\hline 22 & 5.451 & $3.482,31$ & 1.440 & 12.960 \\
\hline 8 & 5.462 & $3.163,29$ & 270 & 14.566 \\
\hline 15 & 5.487 & $2.736,53$ & 1.440 & 11.616 \\
\hline 10 & 5.929 & $5.531,14$ & 1.361 & 27.500 \\
\hline 42 & 6.305 & $5.008,96$ & 588 & 18.117 \\
\hline 9 & 6.615 & $3.302,01$ & 842 & 17.250 \\
\hline 37 & 7.520 & $4.499,07$ & 1.372 & 18.539 \\
\hline 46 & 7.918 & $4.314,94$ & 1.786 & 19.382 \\
\hline 45 & 7.937 & $6.939,63$ & 2.772 & 32.830 \\
\hline 53 & 9.199 & $5.246,77$ & 3.135 & 22.875 \\
\hline 43 & 9.381 & $5.088,14$ & 2.772 & 23.400 \\
\hline 49 & 10.168 & $5.049,43$ & 2.500 & 20.225 \\
\hline 51 & 11.475 & $4.873,97$ & 1.984 & 24.840 \\
\hline 65 & 11.790 & $6.537,18$ & 3.528 & 27.386 \\
\hline 2 & 12.010 & $7.581,35$ & 2.074 & 31.905 \\
\hline 30 & 12.828 & $6.191,22$ & 1.258 & 26.195 \\
\hline 31 & 13.064 & $5.606,87$ & 5.780 & 27.386 \\
\hline 32 & 13.380 & $7.198,12$ & 2.517 & 31.688 \\
\hline 59 & 15.274 & $9.055,95$ & 3.645 & 38.220 \\
\hline 60 & 15.378 & $12.028,36$ & 5.445 & 47.641 \\
\hline 26 & 15.773 & $12.439,61$ & 1.888 & 58.854 \\
\hline 56 & 16.865 & $13.262,8$ & 5.118 & 69.192 \\
\hline 54 & 17.839 & $11.414,8$ & 5.758 & 44.475 \\
\hline 52 & 18.059 & $10.536,16$ & 74 & 37.303 \\
\hline
\end{tabular}




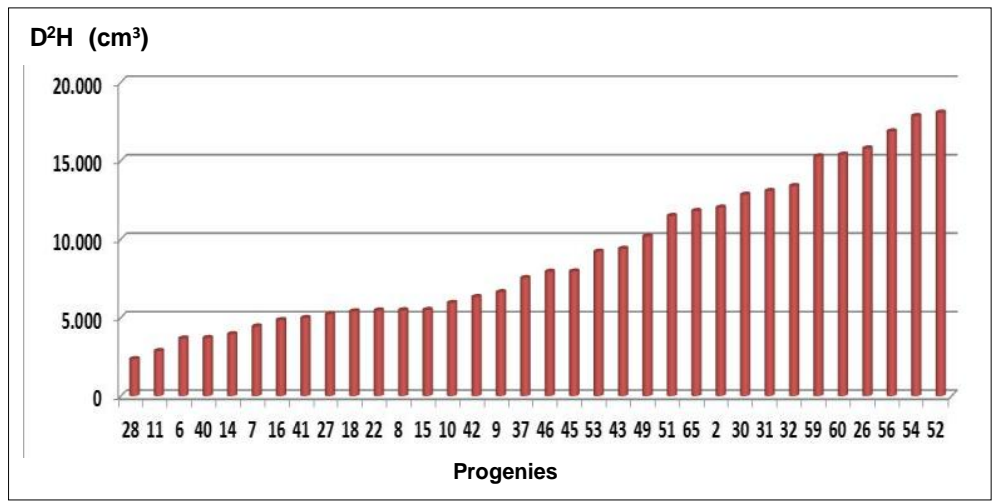

Figura $\mathrm{N}^{\circ} 7$

RESULTADOS EN BIOMASA SEGÚN PROGENIES

Cuadro $\mathrm{N}^{\circ} 10$

ANÁLISIS DE VARIANZA BIOMASA

\begin{tabular}{|l|r|r|r|r|r|}
\hline \multicolumn{1}{|c|}{ FV } & \multicolumn{1}{c|}{ SC } & \multicolumn{1}{c|}{ gl } & \multicolumn{1}{c|}{ CM } & \multicolumn{1}{c|}{ F } & \multicolumn{1}{c|}{ p-valor } \\
\hline Modelo & $4.858 .917,34$ & 786 & $6.181,83$ & 1,65 & 0,2491 \\
\hline Bloque & $538.570,05$ & 24 & $22.440,42$ & 5,99 & 0,0104 \\
\hline Progenie & $538.570,05$ & 33 & $52.173,65$ & 13,92 & 0,0007 \\
\hline Bloque * Progenie & $2.598 .616,84$ & 729 & $3.564,63$ & 0,95 & 0,6067 \\
\hline Error & $26.245,00$ & 7 & $3.749,29$ & & \\
\hline Total & $4.885 .162,34$ & 793 & & & \\
\hline
\end{tabular}


Cuadro $\mathrm{N}^{\circ} 11$

TEST DE COMPARACIÓN DE MEDIAS PARA BIOMASA

\begin{tabular}{|c|c|c|c|c|c|c|c|c|c|}
\hline Progenie & $\begin{array}{c}\text { Medias } \\
D^{2} H_{3}\end{array}$ & \multicolumn{8}{|c|}{ Valor de Comparación` } \\
\hline 28 & 2.349 & A & & & & & & & \\
\hline 11 & 2.866 & A & B & & & & & & \\
\hline 6 & 3.665 & A & B & C & & & & & \\
\hline 40 & 3.695 & A & B & C & & & & & \\
\hline 14 & 3.936 & A & B & C & & & & & \\
\hline 7 & 4.429 & A & B & C & & & & & \\
\hline 16 & 4.835 & A & B & C & & & & & \\
\hline 41 & 4.972 & A & B & C & & & & & \\
\hline 27 & 5.208 & A & B & $\mathrm{C}$ & D & & & & \\
\hline 18 & 5.396 & A & B & $\mathrm{C}$ & D & & & & \\
\hline 22 & 5.451 & A & B & $\mathrm{C}$ & D & & & & \\
\hline 8 & 5.462 & A & B & $\mathrm{C}$ & $\mathrm{D}$ & & & & \\
\hline 15 & 5.487 & A & B & C & D & & & & \\
\hline 10 & 5.929 & A & B & $\mathrm{C}$ & D & $E$ & & & \\
\hline 42 & 6.305 & A & B & $\mathrm{C}$ & $\mathrm{D}$ & $E$ & & & \\
\hline 9 & 6.615 & A & B & C & D & $E$ & & & \\
\hline 37 & 7.520 & A & B & $\mathrm{C}$ & D & $E$ & $\mathrm{~F}$ & & \\
\hline 46 & 7.918 & A & B & $\mathrm{C}$ & D & $E$ & $\mathrm{~F}$ & G & \\
\hline 45 & 7.937 & A & B & C & D & $E$ & $\mathrm{~F}$ & G & \\
\hline 53 & 9.199 & A & B & $\mathrm{C}$ & $\mathrm{D}$ & $E$ & $\mathrm{~F}$ & G & $\mathrm{H}$ \\
\hline 43 & 9.381 & A & B & $\mathrm{C}$ & D & $E$ & $\mathrm{~F}$ & G & $\mathrm{H}$ \\
\hline 49 & 10.168 & A & B & $\mathrm{C}$ & D & $E$ & $F$ & G & $\mathrm{H}$ \\
\hline 51 & 11.475 & A & B & $C$ & D & $E$ & $F$ & G & $\mathrm{H}$ \\
\hline 65 & 11.790 & A & B & C & D & $\mathrm{E}$ & $\mathrm{F}$ & G & $\mathrm{H}$ \\
\hline 2 & 12.010 & A & B & $C$ & D & $E$ & $F$ & G & $\mathrm{H}$ \\
\hline 30 & 12.828 & & B & $C$ & D & $E$ & $F$ & G & $\mathrm{H}$ \\
\hline 31 & 13.064 & & & C & D & $E$ & $\mathrm{~F}$ & G & $\mathrm{H}$ \\
\hline 32 & 13.380 & & & $C$ & D & $E$ & $F$ & G & $\mathrm{H}$ \\
\hline 59 & 15.274 & & & & D & $E$ & $\mathrm{~F}$ & G & $\mathrm{H}$ \\
\hline 60 & 15.378 & & & & D & $E$ & $F$ & G & $\mathrm{H}$ \\
\hline 26 & 15.773 & & & & & $E$ & $F$ & G & $\mathrm{H}$ \\
\hline 56 & 16.865 & & & & & & $F$ & G & $\mathrm{H}$ \\
\hline 54 & 17.839 & & & & & & & G & $\mathrm{H}$ \\
\hline 52 & 18.059 & & & & & & & & $\mathrm{H}$ \\
\hline
\end{tabular}




\section{Supervivencia}

En la supervivencia que registra el ensayo a los 33 meses de edad es alta, excepto dos progenies, los valores están entre 80 y $100 \%$, y 22 de ellas muestran valores por sobre el $90 \%$ (Cuadro $\mathrm{N}^{\circ} 12$ y Figura $\mathrm{N}^{\circ} 8$ ).

Cuadro $\mathrm{N}^{\circ} 12$

RESULTADOS DE SUPERVIVENCIA SEGÚN PROGENIES

\begin{tabular}{|c|c|}
\hline Progenie & $\begin{array}{c}\text { Supervivencia } \\
(\%)\end{array}$ \\
\hline 6 & 68 \\
\hline 8 & 72 \\
\hline 43 & 80 \\
\hline 53 & 80 \\
\hline 60 & 84 \\
\hline 49 & 84 \\
\hline 45 & 84 \\
\hline 40 & 84 \\
\hline 28 & 84 \\
\hline 37 & 88 \\
\hline 59 & 88 \\
\hline 15 & 88 \\
\hline 52 & 92 \\
\hline 56 & 92 \\
\hline 31 & 92 \\
\hline 30 & 92 \\
\hline 32 & 92 \\
\hline 2 & 92 \\
\hline 65 & 92 \\
\hline 70 & 92 \\
\hline 42 & 92 \\
\hline 18 & 92 \\
\hline 16 & 92 \\
\hline 41 & 96 \\
\hline 11 & 96 \\
\hline 10 & 96 \\
\hline 7 & 92 \\
\hline 14 & 92 \\
\hline 27 & 98 \\
\hline 54 & 100 \\
\hline 26 & 100 \\
\hline 51 & 100 \\
\hline 46 & 100 \\
\hline 22 & 100 \\
\hline
\end{tabular}




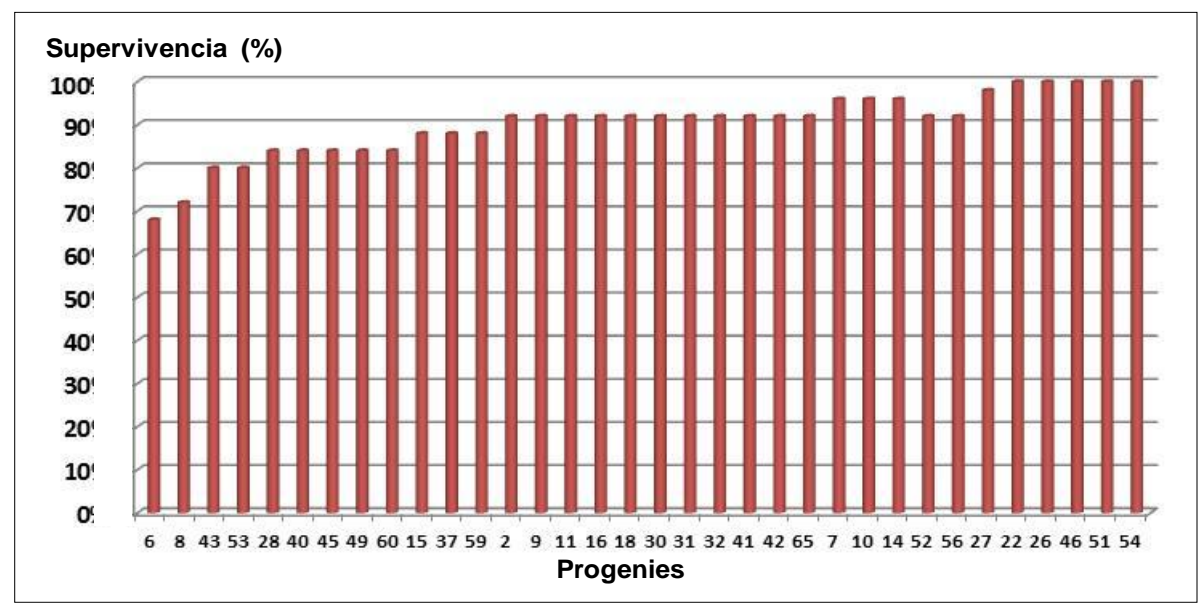

Figura $\mathbf{N}^{\circ} 8$

RESULTADOS EN SUPERVIVENCIA SEGÚN PROGENIES

\section{DISCUSIÓN}

Se efectúa primero una comparación de las variables respuesta obtenidas en la primera evaluación (2013) y aquellas que arroja la segunda evaluación (2014), se hace después un análisis general del ensayo y se muestra posteriormente un modelo desarrollado para la estimación de la altura a partir del DAP.

\section{Comparación Evaluaciones 2013 y 2014}

De los resultados obtenidos en ambas evaluaciones se desprende en general que estos son muy positivos, se registran buenos resultados para las variables altura y diámetro, en consecuencias también para el estimador de biomasa, y se aprecia igualmente una alta supervivencia que se mantiene en la segunda evaluación. Esto indica que la especie registra un buen crecimiento y una buena adaptación, en especial si se recuerda que las progenies probadas provienen de zonas tan dispares como las regiones del Maule (Parral) y Los Ríos (Valdivia).

La comparación de los valores obtenidos para las variables diámetro, altura, estimador de biomasa y supervivencia en ambas evaluaciones indica que las tendencias se mantienen en los resultados de las diferentes progenies y que el ensayo podrá ser una buena fuente de material de propagación para apoyar en materia de un mejoramiento genético posterior.

En el caso del DAP, se aprecia que se mantienen en la segunda evaluación las tendencias manifestadas por las distintas progenies en la evaluación anterior y se observan para esta variable un incremento promedio por árbol de $1,5 \mathrm{~cm}$ por año e incrementos máximos de 3,9 $\mathrm{cm}$ por año. Al observar los resultados según progenies, se destacan los incrementos medios registrados por las progenies 54 y 56 con 2,0 y 2,1 cm, respectivamente. Ambas progenies son las que presentan los mayores registros para esta variable en el ensayo (Cuadro $\mathrm{N}^{\circ} 1$ y Figura $\mathrm{N}^{\circ} 1$ de Anexo).

En cuanto a las alturas, también se mantienen las tendencias observadas en la primera 
evaluación y se aprecian un incremento promedio por árbol de $1,5 \mathrm{~m}$ e incrementos máximos cercanos a los $4 \mathrm{~m}$. Destacan los incrementos registrados por las progenies 54 y 56 con 1,9 y 1,8 metros, respectivamente. Ambas progenies son las que presentan los mayores registros para esta variable en el ensayo (Cuadro $\mathrm{N}^{\circ} 2$ y Figura $\mathrm{N}^{\circ} 2$ de Anexo).

Respecto del estimador de biomasa, igualmente se mantienen las tendencias y destacan los valores de incremento de esta variable en las progenies 54 y 52 (Cuadro $N^{\circ} 3$ y Figura $N^{\circ} 3$ de Anexo).

La supervivencia en tanto es similar a la registrada el año 2013, llegando a un promedio del $90 \%$. Destaca que el $88 \%$ de las progenies presentan una supervivencia igual o mayor al $80 \%$ y de ellas $(22,26,46,51$ y 54$)$ se mantienen en un $100 \%$ después de 33 meses (Cuadro $N^{\circ} 4$ y Figura $\mathrm{N}^{\circ} 4$ de Anexo).

\section{Evaluación General del Ensayo}

En general las procedencias y progenies evaluadas en el ensayo presentan distintos niveles de desarrollo. La relación entre DAP y altura media registrada en las distintas progenies a los 33 meses de edad se presenta en la Figura $\mathrm{N}^{\circ}$ 9. Se observa un desarrollo lineal, correspondiente al crecimiento inicial de las progenies.

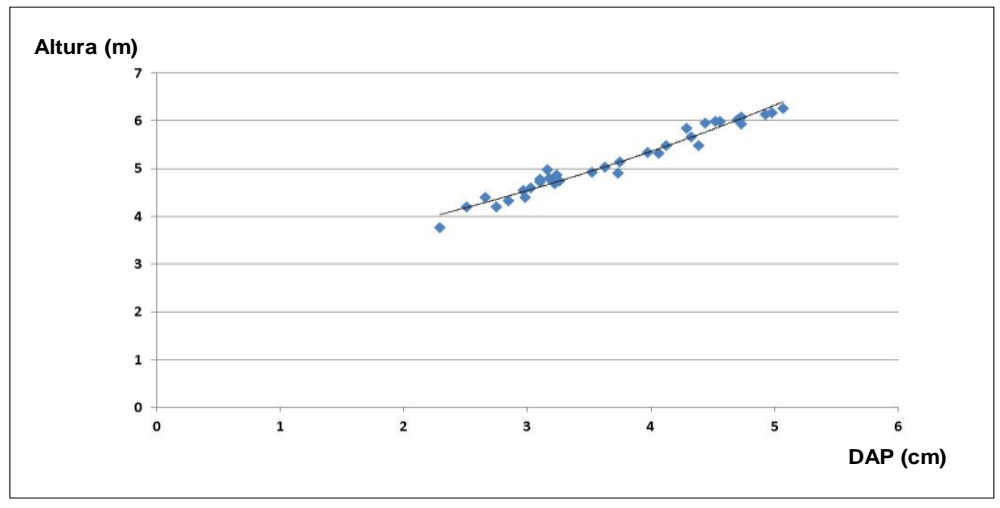

Figura $N^{\circ} 9$

RELACIÓN DAP-ALTURA PROMEDIOS DE LAS PROGENIES

De acuerdo a los resultados obtenidos a los 33 meses de instalado el ensayo, el mayor valor obtenido en el DAP corresponde a una procedencia Mafil (Región de Los Ríos), una de Retiro (Región del Maule) y una de El Rosal, Yumbel (Región del Biobio), con valores cercanos a los $5 \mathrm{~cm}$. Estas mismas progenies ya habían presentado los mejores resultados en la evaluación del año 2013.

En general estos resultados en crecimiento en DAP son similares a los obtenidos a anteriormente, indicando que estas progenies están respondiendo bien a las condiciones locales del ensayo de progenies en Los Sauces.

Es así como la progenie MR-54 Mafil (Región de Los Ríos), presenta diferencias significativas con las progenies restantes, tanto para la variable DAP como para altura y $D^{2} \mathrm{H}$, además, esta progenie presenta, un $100 \%$ de supervivencia en el ensayo. Por el contrario, el menor valor en DAP lo presentó una familia originada desde un ensayo en Antiquina (MR-28). 
Al considerar los 12 mejores resultados del ensayo en términos de DAP, se observa que las procedencias Mafil y Retiro aportan con 4 y 3 familias, respectivamente. Otras procedencias destacadas corresponden Antiquina, Florida y Yumbel, las que contribuyen con el mejor material en el ensayo. Resultados semejantes fueron registrados en la evaluación 2013.

En términos de la altura, variable más relacionada con el sitio, familias de Mafil y Yumbel, presentaron los mayores valores a los 33 meses de instalado el ensayo, con valores de 6,3 y 6,2 m, respectivamente.

Otras procedencias y progenies de mejor crecimiento en altura son las ya destacadas en el caso del DAP y corresponden a progenies obtenidas desde 3 árboles en Mafil, 3 en Retiro y 1 en Antiquina.

Al considerar las 10 mejores resultados en altura, la procedencia Máfil nuevamente ubica a 4 de sus familias dentro de las mejores del ensayo.

Otras buenas procedencias corresponden a 3 familias de Retiro, dos de Yumbel y una de Antiquina, las que contribuyen con el mejor material en el ensayo. El menor valor en altura nuevamente lo presentó una familia de Antiquina (MR-28).

En relación a la estimación de biomasa, D²H, y al igual que en la evaluación del año 2013 , una familia de la procedencia Yumbel presenta el mayor valor del ensayo. Otras procedencias de desempeño superior nuevamente corresponden a Mafil, Retiro y Antiquina, presentando los mayores valores del ensayo.

Al considerar las 10 mejores resultados en la estimación de biomasa, la procedencia Máfil nuevamente presenta la mayor cantidad de familias, ubicando en este caso a 4 de sus progenies entre ellas. El listado se completa con 3 familias del ensayo de progenies en Retiro, 2 familias de Antiquina y una familia de Yumbel. El menor rendimiento en este estimador fue presentado por la familia MR-28 de Antiquina.

Los resultados obtenidos señalan una tendencia que se manifiesta en forma constante en el ensayo a los 33 meses de su instalación. Sin embargo, la alta variabilidad de la especie observada en terreno y su falta de domesticación se manifiesta claramente a través de las diferencias existentes entre familias y más aún, entre una misma procedencia. Ello señala la conveniencia de avanzar hacia el mejoramiento genético de la especie.

En términos de supervivencia, 5 familias presentan un $100 \%$ en esta variable y en total 22 familias presentan un valor mayor al $90 \%$. Cabe agregar que de las familias ensayadas, un $94 \%$ (32) presenta una supervivencia del $80 \%$ o más.

Las 5 familias con un 100 de supervivencia corresponden a 2 progenies de la procedencia Antiquina, 1 de Yumbel, 1 una de Mafil, y 1 progenie proveniente de semilla comercial de Australia. El menor valor lo presentó la progenie MR-6 de Antiquina con un valor del $68 \%$ de supervivencia.

Al analizar el comportamiento de las familias empleando las 10 mejores para cada variable evaluada, se destacan 8 familias que coinciden con los mejores resultados (Cuadro $\mathrm{N}^{\circ} 13$ ).

Se destacan 4 familias de Máfil, 2 de Retiro, 1 de Yumbel y 1 de Antiquina. Estas progenies son las que han presentado el mejor comportamiento transcurridos 33 meses desde la instalación del ensayo. 
Cuadro $\mathrm{N}^{\circ} 13$

MEJORES FAMILIAS A LOS 33 MESES DE EDAD

\begin{tabular}{|c|l|c|}
\hline Código & \multicolumn{1}{|c|}{ Procedencia } & Progenie \\
\hline MR-52 & Yumbel. Región del Bío Bío & Plus 1 \\
\hline MR-54 & Mafil. Valdivia, Región de Los Ríos & Plus 6 \\
\hline MR-56 & Retiro. Región del Maule & Plus 1 \\
\hline MR-26 & Antiquina. Cañete, Región del Bío Bío & Árbol 6 \\
\hline MR-59 & Retiro. Región del Maule & Plus 4 \\
\hline MR-31 & Mafil. Valdivia, Región de Los Ríos & Plus 3 \\
\hline MR-32 & Mafil. Valdivia, Región de Los Ríos & Plus 4 \\
\hline MR-30 & Mafil. Valdivia, Región de Los Ríos & Plus 2 \\
\hline
\end{tabular}

Otro aspecto interesante es que en general todas las procedencias incluidas en el ensayo realizan un aporte en árboles individuales registrándose una alta variación dentro de una misma familia o progenie.

Lo anterior indica claramente que podrían seleccionarse árboles individuales para conformar una segunda generación o bien una unidad de producción de semilla mejorada para avanzar hacia un programa de mejoramiento genético con la especie.

Este conjunto de antecedentes es importante además porque constituyen la primera información generada en la región de La Araucanía respecto de procedencias y progenies de esta especie.

\section{Modelo de Regresión DAP-Altura} al DAP.

Con la información reunida se desarrolló un modelo juvenil de estimación de alturas en base

Este modelo corresponde a:

$$
H(m)=2,35+0,71^{*} \text { DAP }(\mathrm{cm}) \quad R^{2}=0,7
$$

El desarrollo del modelo fue respaldado por un análisis de varianza que permitió definir una bondad de ajuste de $R^{2}=0,7$.

El modelo así puede ser utilizado en forma segura y en la Figura $\mathrm{N}^{\circ} 10$ se indica gráficamente la correspondencia entre los datos de campo y los estimados con el modelo juvenil DAPAltura. 


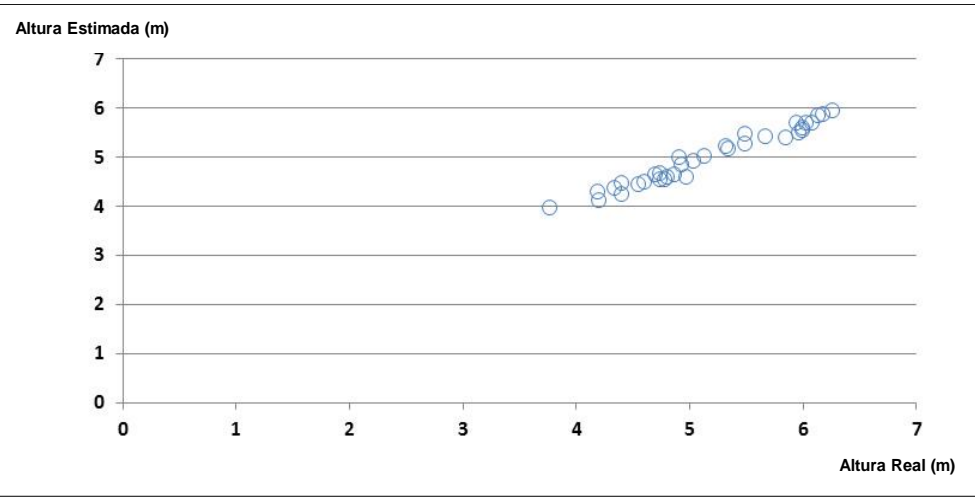

Figura $N^{\circ} 10$

DATOS DE CAMPO Y DATOS ESTIMADOS PARA LA ALTURA

\section{CONCLUSIONES}

La sobrevivencia obtenida en el ensayo es en general mayor al 80\%, con 22 de 34 familias presentando una valor superior al $90 \%$ y con 32 de las 34 familias presentando una supervivencia mayor al o igual al $80 \%$, valores que se sitúan en lo esperado para este tipo de especies.

La mejor procedencia/progenie con diferencias significativas en las variables evaluadas respecto de las demás fue MR-52 de Yumbel, tanto en la medición de 2013 como en la de 2014.

De acuerdo a los resultados obtenidos, las mejores 8 progenies a los 33 meses son:

$\begin{array}{ll}\text { MR-52 } & \text { Yumbel. Región del Bío Bío } \\ \text { MR-54 } & \text { Mafil. Valdivia, Región de Los Ríos } \\ \text { MR-56 } & \text { Retiro. Región del Maule } \\ \text { MR-26 } & \text { Antiquina. Cañete, Región del Bío Bío } \\ \text { MR-59 } & \text { Retiro. Región del Maule } \\ \text { MR-31 } & \text { Mafil. Valdivia, Región de Los Ríos } \\ \text { MR-32 } & \text { Mafil. Valdivia, Región de Los Ríos } \\ \text { MR-30 } & \text { Mafil. Valdivia, Región de Los Ríos }\end{array}$

Las procedencias de Máfil, Antiquina, Retiro y Yumbel presentan los mejores resultados, los que deben ser también ser relacionados con las condiciones de suelo y clima presentes en el área del ensayo, de modo de poder hacerlos extensibles hacia sitios semejantes en la región.

Las diferencias detectadas entre procedencias y progenies a los 33 meses de instalado el ensayo, señala claramente la alta variabilidad de la especie y su falta de domesticación a nivel local, destacando que todas las procedencias utilizadas en el ensayo, realizan un aporte en árboles individuales.

Los valores para los árboles dentro de una misma familia o progenie tienen una alta variación, lo que indica claramente que podrían seleccionarse más adelante árboles individuales para conformar una segunda generación o bien una unidad de producción de semilla mejorada, lo que indica la necesidad de avanzar en programas de mejoramiento genético de la especie. 
Los resultados presentados deben ser ratificados a través de evaluaciones posteriores, siendo aún no concluyentes.

Este conjunto de antecedentes es importante dado que es la primera información sobre la especie para la región de La Araucanía.

La definición de individuos o familias que pasarían a la segunda generación para avanzar hacia el mejoramiento genético deberá apoyarse con un análisis BLUP y esto requeriría esperar hasta al menos la mitad de la rotación estimada para la especie, que se estima en principio en12 años. Muy probablemente será conveniente la incorporación de nuevos genotipos a través de una población de infusión de modo de aumentar la intensidad de selección.

\section{RECONOCIMIENTOS}

Se agradece a Bosques Cautín SA su valioso apoyo y las facilidades otorgadas para la instalación y evaluación del ensayo.

\section{REFERENCIAS}

Correa, M.; Toloza, R.; Pereyra, L.; Silva, F. y FriedI, R., 2011. Resultados iniciales de un ensayo de acacia negra (Acacia mearnsii De Wild) en la provincia de Misiones. XIII Jornadas Técnicas Forestales y Ambientales. Facultad de Ciencias Forestales, UNaM - EEA Montecarlo, INTA. El Dorado, Misiones, Argentina.

Higa, A. R.; Dedecek, R. A.; Dos Santos, A. F.; Stein, P. P. y Simon, A. A., 1998. Desarrollo de sistemas de producción para acacia negra (Acacia mearnsii De Wild.). Resumen.

INFOR, 1998. Incorporación de especies del género Acacia a la producción forestal chilena. Formulario de presentación proyecto FDI. INFOR-CORFO. Santiago 150 p.

INFOR, 1999. Incorporación de especies del género Acacia a la producción forestal chilena. Informe de avance. INFOR - CORFO. Concepción. 126 p.

Kannegiesser, U., 1990. Apuntes sobre algunas acacias australianas: 1. Acacia mearnsii De Willd. Ciencia e Investigación Forestal, 4 (2):198-212.

Pinilla, J. C., 2000. Descripción y antecedentes básicos sobre Acacia dealbata, A. melanoxylon y $A$. mearnsii. Informe Técnico №147. INFOR-CORFO. 49p.

Pinilla, J. C.; Molina M. P. y Gutiérrez, B., 2004. Acacia en el Desarrollo Forestal Productivo de Chile: Avances de Investigación. En: Actas Seminario Diversificación Forestal: Nuevas opciones, nuevos productos, nuevos mercados. INFOR. Villarrica, Chile. 13-15 Octubre 2004

Pinilla, J. C.; Molina, M. P.; Hernández, G.; Barros, S.; Ortiz, O. y Navarrete, M., 2011. Avances de la investigación con especies del género Acacia en Chile. Informe Técnico N¹79. Instituto Forestal, Sede Bio Bio, Chile.

Pinilla S., J. C.; Navarrete, M., 2013. Antecedentes del crecimiento de Acacia mearnsii de Wild según distintas densidades iniciales para dos sitios en la región del Biobío [artículos de revistas]. 2013, Ago.. Publicado en: Ciencia e Investigación Forestal, CIFOR, v.19:n2, Pág.: 71-92

Searle, S. D., 2000. Black Wattle (Acacia mearnsii) for farm forestry. Department of Natural Resource and Environment. Victoria. 18p.

Turnbull, J. W., 1986. Multipurpose Australian trees and shrubs. Lesser known species for fuelwood and agroforestry: ACIAR Monograph Num. 1, 316 p. 
RESULTADOS PRIMERA (2013) Y SEGUNDA (2014) EVALUACIÓN

Cuadro $\mathrm{N}^{\circ} 1$

DIÁMETROS POR PROGENIE EVALUACIONES 2013 Y 2014

\begin{tabular}{|c|c|c|}
\hline \multirow{3}{*}{ Progenies } & \multicolumn{2}{|c|}{ DAP } \\
\hline & 2013 & 2014 \\
\hline & \multicolumn{2}{|c|}{ (cm) } \\
\hline 2 & 2,68 & 4,39 \\
\hline 6 & 1,51 & 2,66 \\
\hline 7 & 1,69 & 2,97 \\
\hline 8 & 1,92 & 3,22 \\
\hline 9 & 2,11 & 3,52 \\
\hline 10 & 1,86 & 3,16 \\
\hline 11 & 1,43 & 2,51 \\
\hline 14 & 1,57 & 2,85 \\
\hline 15 & 1,91 & 3,26 \\
\hline 16 & 1,76 & 3,03 \\
\hline 18 & 1,76 & 3,10 \\
\hline 22 & 1,85 & 3,18 \\
\hline 26 & 2,91 & 4,70 \\
\hline 27 & 1,78 & 3,10 \\
\hline 28 & 1,16 & 2,29 \\
\hline 30 & 2,79 & 4,44 \\
\hline 31 & 2,85 & 4,56 \\
\hline 32 & 2,72 & 4,52 \\
\hline 37 & 2,11 & 3,63 \\
\hline 40 & 1,43 & 2,75 \\
\hline 41 & 1,63 & 2,98 \\
\hline 42 & 1,84 & 3,24 \\
\hline 43 & 2,38 & 4,06 \\
\hline 45 & 2,07 & 3,73 \\
\hline 46 & 2,11 & 3,75 \\
\hline 49 & 2,38 & 4,12 \\
\hline 51 & 2,53 & 4,29 \\
\hline 52 & 3,20 & 4,98 \\
\hline 53 & 2,34 & 3,97 \\
\hline 54 & 3,06 & 5,07 \\
\hline 56 & 2,88 & 4,93 \\
\hline 59 & 2,82 & 4,73 \\
\hline 60 & 2,94 & 4,73 \\
\hline 65 & 2,63 & 4,33 \\
\hline
\end{tabular}




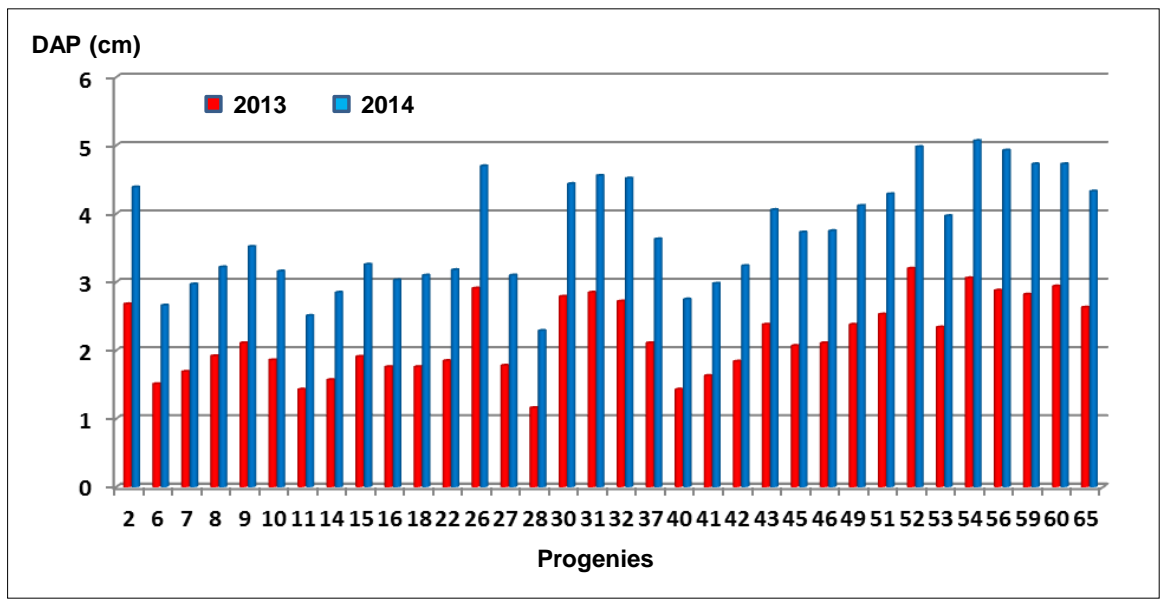

Figura $\mathrm{N}^{\circ} 1$

DIÁMETROS POR PROGENIE EVALUACIONES 2013 Y 2014 


\section{Cuadro $\mathrm{N}^{\circ} 2$}

ALTURAS POR PROGENIE EVALUACIONES 2013 Y 2014

\begin{tabular}{|c|c|c|}
\hline \multirow{3}{*}{ Progenies } & \multicolumn{2}{|c|}{ Altura } \\
\hline & 2013 & 3014 \\
\hline & \multicolumn{2}{|c|}{ (m) } \\
\hline 2 & 4,00 & 5,49 \\
\hline 6 & 2,98 & 4,40 \\
\hline 7 & 3,30 & 4,55 \\
\hline 8 & 3,37 & 4,69 \\
\hline 9 & 3,31 & 4,92 \\
\hline 10 & 3,56 & 4,97 \\
\hline 11 & 2,99 & 4,20 \\
\hline 14 & 3,19 & 4,33 \\
\hline 15 & 3,50 & 4,74 \\
\hline 16 & 3,39 & 4,60 \\
\hline 18 & 3,33 & 4,73 \\
\hline 22 & 3,41 & 4,80 \\
\hline 26 & 4,44 & 6,02 \\
\hline 27 & 3,44 & 4,78 \\
\hline 28 & 2,69 & 3,76 \\
\hline 30 & 4,27 & 5,96 \\
\hline 31 & 4,20 & 5,99 \\
\hline 32 & 4,17 & 5,99 \\
\hline 37 & 3,50 & 5,03 \\
\hline 40 & 3,00 & 4,19 \\
\hline 41 & 3,04 & 4,40 \\
\hline 42 & 3,37 & 4,86 \\
\hline 43 & 3,74 & 5,32 \\
\hline 45 & 3,59 & 4,90 \\
\hline 46 & 3,71 & 5,13 \\
\hline 49 & 3,78 & 5,49 \\
\hline 51 & 4,10 & 5,84 \\
\hline 52 & 4,52 & 6,17 \\
\hline 53 & 3,77 & 5,34 \\
\hline 54 & 4,40 & 6,26 \\
\hline 56 & 4,30 & 6,13 \\
\hline 59 & 4,40 & 6,08 \\
\hline 60 & 4,43 & 5,94 \\
\hline 65 & 4,03 & 5,66 \\
\hline
\end{tabular}




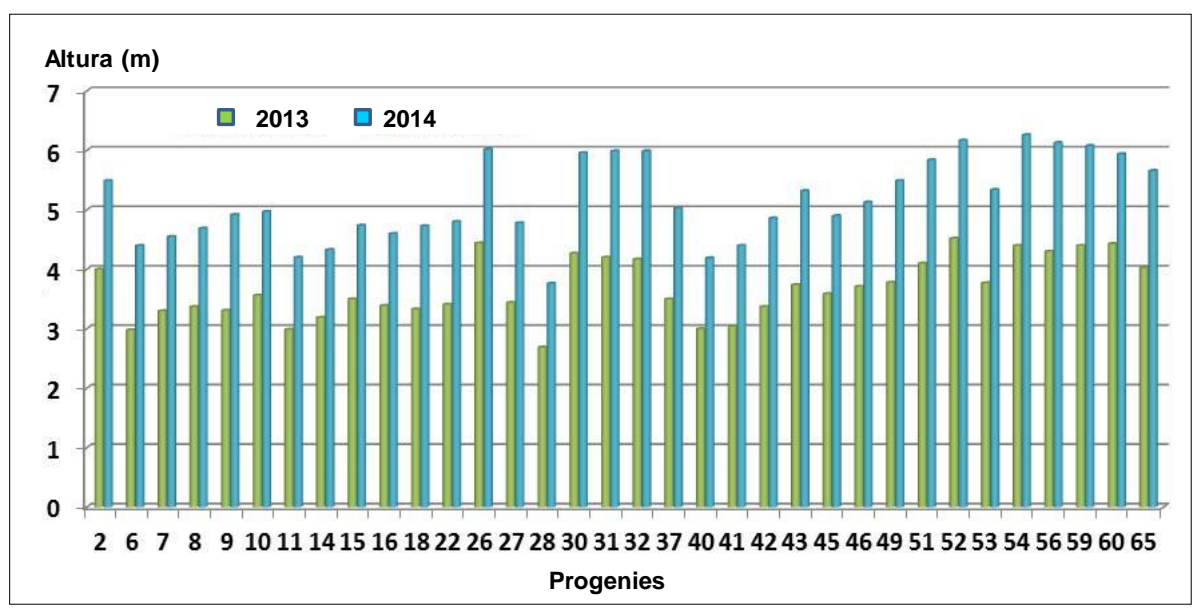

Figura $\mathrm{N}^{\circ} 2$

ALTURAS POR PROGENIE EVALUACIONES 2013 Y 2014 


\section{Cuadro $\mathrm{N}^{\circ} 3$}

BIOMASA POR PROGENIE EVALUACIONES 2013 Y 2014

\begin{tabular}{|c|c|c|}
\hline \multirow{3}{*}{ Progenies } & \multicolumn{2}{|c|}{ Indicador Biomasa $\left(D^{2} H\right)$} \\
\hline & 2013 & 2014 \\
\hline & \multicolumn{2}{|c|}{$\left(\mathrm{cm}^{3}\right)$} \\
\hline 28 & 5,73 & 23,50 \\
\hline 11 & 7,56 & 28,66 \\
\hline 6 & 7,76 & 36,65 \\
\hline 40 & 8,01 & 36,95 \\
\hline 14 & 10,25 & 39,36 \\
\hline 7 & 10,97 & 44,28 \\
\hline 16 & 12,95 & 48,34 \\
\hline 41 & 11,08 & 49,72 \\
\hline 27 & 13,60 & 52,08 \\
\hline 18 & 14,40 & 53,96 \\
\hline 22 & 14,13 & 54,51 \\
\hline 8 & 14,34 & 54,62 \\
\hline 15 & 14,39 & 54,87 \\
\hline 10 & 15,03 & 59,28 \\
\hline 42 & 16,38 & 63,04 \\
\hline 9 & 16,57 & 66,16 \\
\hline 37 & 19,65 & 75,20 \\
\hline 46 & 18,90 & 79,18 \\
\hline 45 & 20,25 & 79,37 \\
\hline 53 & 23,93 & 91,98 \\
\hline 43 & 25,58 & 93,82 \\
\hline 49 & 24,09 & 101,68 \\
\hline 51 & 28,29 & 114,76 \\
\hline 65 & 33,28 & 117,90 \\
\hline 2 & 33,98 & 120,10 \\
\hline 30 & 38,31 & 128,29 \\
\hline 31 & 38,06 & 130,65 \\
\hline 32 & 35,87 & 133,80 \\
\hline 59 & 40,72 & 152,74 \\
\hline 60 & 46,96 & 153,8 \\
\hline 26 & 48,58 & 157,73 \\
\hline 56 & 42,82 & 168,65 \\
\hline 54 & 49,1 & 178,41 \\
\hline 52 & 52,04 & 180,59 \\
\hline
\end{tabular}




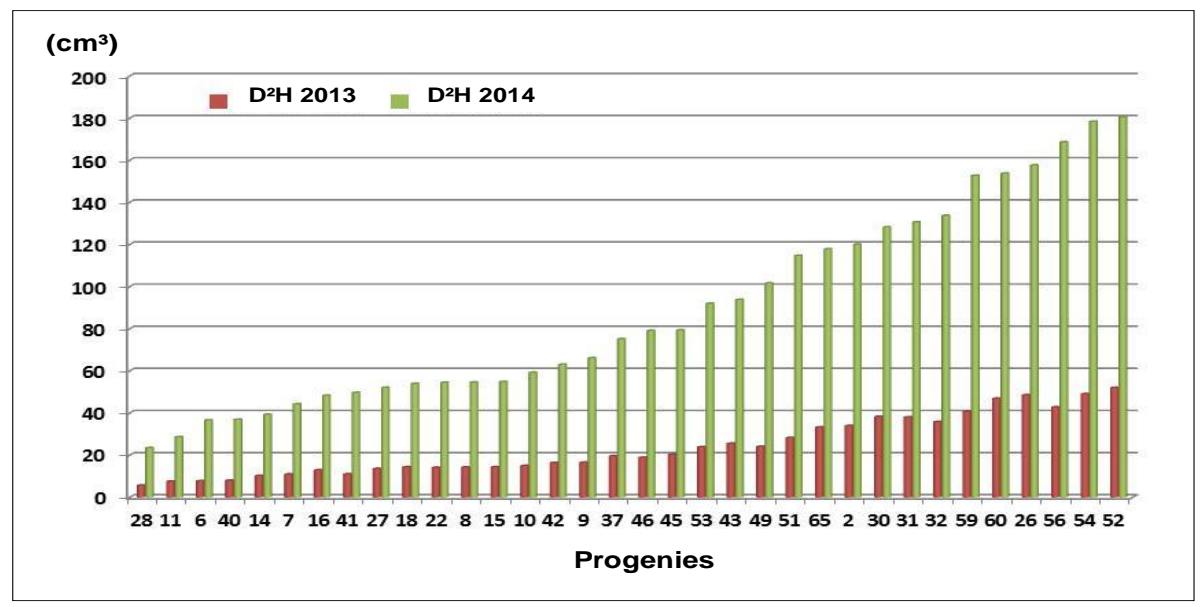

Figura $\mathbf{N}^{\circ} 3$

BIOMASA POR PROGENIE EVALUACIONES 2013 Y 2014 
Cuadro $\mathrm{N}^{\circ} 4$

SUPERVIVENCIA POR PROGENIE EVALUACIONES 2013 Y 2014

\begin{tabular}{|c|c|c|}
\hline \multirow{3}{*}{ Progenies } & \multicolumn{2}{|c|}{ Supervivencia } \\
\hline & 2013 & 2014 \\
\hline & & (\%) \\
\hline 6 & 68 & 68 \\
\hline 8 & 72 & 72 \\
\hline 43 & 80 & 80 \\
\hline 53 & 80 & 80 \\
\hline 28 & 84 & 84 \\
\hline 40 & 84 & 84 \\
\hline 45 & 84 & 84 \\
\hline 49 & 84 & 84 \\
\hline 60 & 84 & 84 \\
\hline 15 & 88 & 88 \\
\hline 37 & 88 & 88 \\
\hline 59 & 88 & 88 \\
\hline 2 & 92 & 92 \\
\hline 9 & 92 & 92 \\
\hline 11 & 92 & 92 \\
\hline 16 & 92 & 92 \\
\hline 18 & 92 & 92 \\
\hline 30 & 92 & 92 \\
\hline 31 & 92 & 92 \\
\hline 32 & 92 & 92 \\
\hline 41 & 92 & 92 \\
\hline 42 & 92 & 92 \\
\hline 65 & 92 & 92 \\
\hline 7 & 96 & 96 \\
\hline 10 & 96 & 96 \\
\hline 14 & 96 & 96 \\
\hline 52 & 92 & 92 \\
\hline 56 & 92 & 92 \\
\hline 27 & 98 & 98 \\
\hline 22 & 100 & 100 \\
\hline 26 & 100 & 100 \\
\hline 46 & 100 & 100 \\
\hline 51 & 100 & 100 \\
\hline 54 & 100 & 100 \\
\hline
\end{tabular}




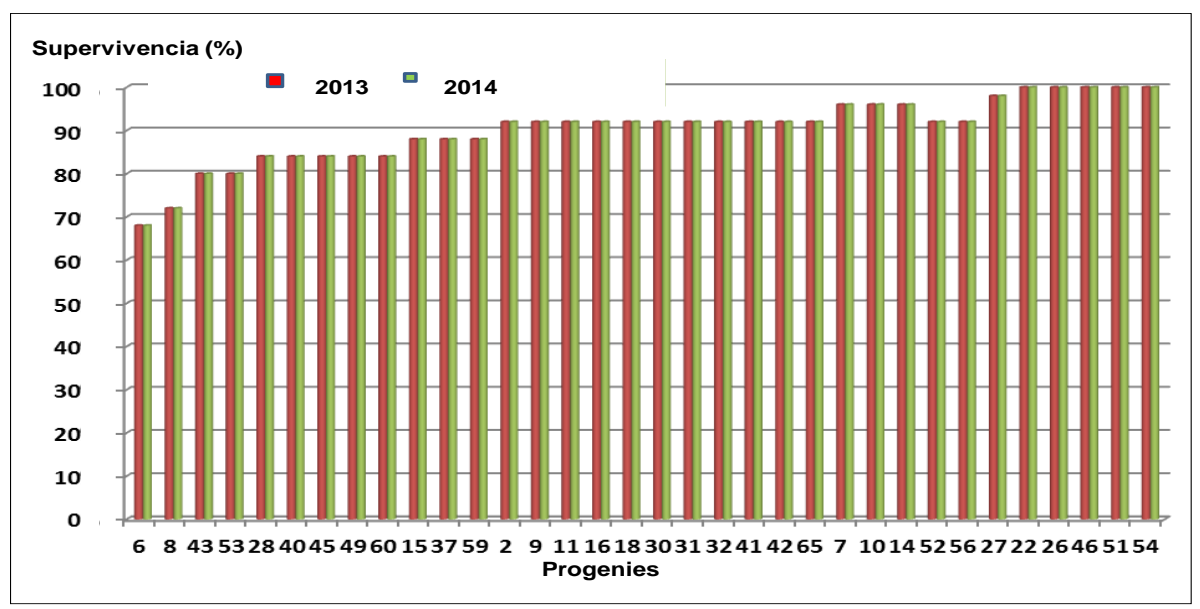

Figura $\mathbf{N}^{\circ} 4$

SUPERVIVENCIA POR PROGENIE EVALUACIONES 2013 Y 2014 